\title{
Eruptive Annular Papules on the Trunk of an Organ Transplant Recipient
}

Abdullah Aleisa, MD; David S. Feingold, MD; Julie Y. Tse, MD

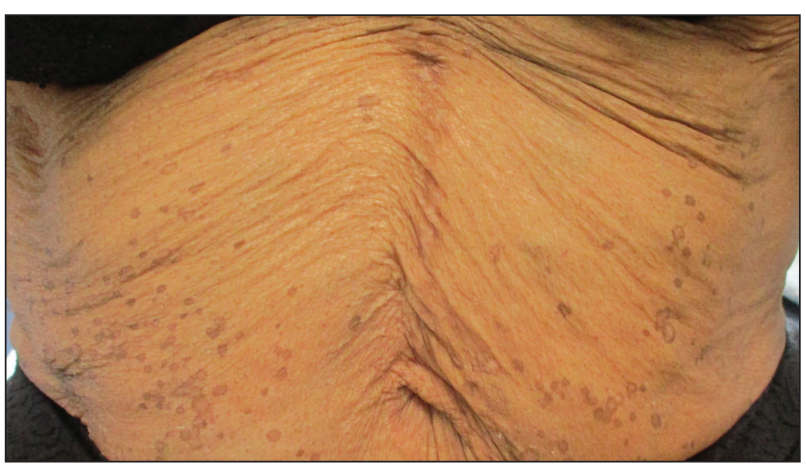

A 50-year-old Black woman with systemic lupus erythematosus and a renal transplant 15 years prior due to lupus nephritis presented with a nonpruritic rash on the abdomen of 1 year's duration. Her immunosuppressive regimen consisted of tacrolimus, azathioprine, and prednisone. Physical examination revealed numerous monomorphic, annular, hyperpigmented, and thin papules with central clearing present on the abdomen extending to the flanks and groin. The patient denied any family history of similar lesions. A 4-mm punch biopsy of an abdominal lesion was performed.

\section{WHAT'S YOUR DIAGNOSIS?}
a. cutaneous lupus erythematosus
b. epidermodysplasia verruciformis
c. porokeratosis
d. syphilis
e. tinea versicolor

From Tufts Medical Center, Tufts University School of Medicine, Boston, Massachusetts. Drs. Aleisa and Feingold are from the Department of Dermatology, and Dr. Tse is from the Department of Pathology.

The authors report no conflict of interest.

Correspondence: Abdullah Aleisa, MD, Department of Dermatology, Tufts Medical Center, Tufts University School of Medicine, 260 Tremont St, 14th Floor, Boston, MA 02116 (abdullah213@gmail.com). 


\section{THE DIAGNOSIS: Epidermodysplasia Verruciformis}

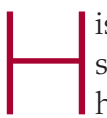
istopathologic examination of our patient's biopsy specimen revealed mild acanthosis with prominent hypergranulosis and enlarged keratinocytes with blue-gray cytoplasm (Figure). A diagnosis of acquired epidermodysplasia verruciformis (EV) was rendered. The patient was treated with photodynamic therapy utilizing 5-aminolevulinic acid.

Epidermodysplasia verruciformis is characterized by susceptibility to human papillomavirus (HPV) infections via a defect in cellular immunity. Epidermodysplasia verruciformis was first described as an autosomal-recessive genodermatosis, but it can be acquired in immunosuppressed states with an atypical clinical appearance. ${ }^{1}$ There are few case reports in skin of color. Acquired EV appears in patients with acquired immunodeficiencies that are susceptible to EV-causing HPVs via a similar mechanism found in inherited EV. ${ }^{2}$ The most common HPV serotypes involved in EV are HPV- 5 and HPV- 8 . The duration of immunosuppression has been found to be positively correlated with the risk for EV development, with the majority of patients developing lesions after 5 years of immunosuppression. ${ }^{3}$ There is an approximately $60 \%$ risk of malignant transformation of EV lesions into nonmelanoma skin cancer. ${ }^{2}$ This risk is believed to be lower in patients with darker skin.4

Preventative measures including sun protection and annual surveillance are crucial in EV patients given the high rate of malignant transformation in sun-exposed lesions. ${ }^{5}$ Treatment options for $\mathrm{EV}$ are anecdotal and have variable results, ranging from topicals including 5-fluorouracil and imiquimod to systemic medications including acitretin and interferon. ${ }^{3}$ Photodynamic therapy can be used for extensive EV. Surgical modalities and other destructive methods also have been tried. ${ }^{6}$

Epidermodysplasia verruciformis often can be confused with similar dermatoses. Porokeratosis appears as annular pink papules with waferlike peripheral scales. Tinea versicolor is a dermatophyte infection caused by Malassezia furfur and presents as multiple dyspigmented, finely scaling, thin papules and plaques. Subacute cutaneous lupus erythematosus presents as pink, scaly,

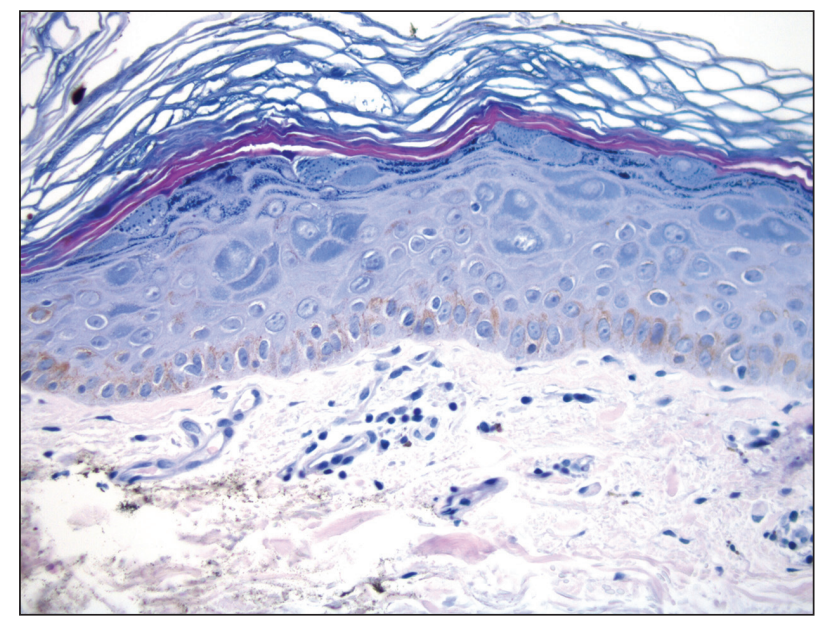
Acanthosis with prominent hypergranulosis and enlarged keratinocytes with blue-gray cytoplasm ( $\mathrm{H} \& \mathrm{E}$, original magnification $\times 40$ ).

annular or psoriasiform papules and plaques most commonly on the trunk. Discoid lupus erythematosus presents as pink, hypopigmented or depigmented, atrophic plaques with a peripheral rim of erythema that indicates activity. Secondary syphilis, commonly denoted as the "great mimicker," presents as psoriasiform papules and plaques among other variable morphologies.

\section{REFERENCES}

1. Sa NB, Guerini MB, Barbato MT, et al. Epidermodysplasia verruciformis: clinical presentation with varied forms of lesions. An Bras Dermatol. 2011;86(4 suppl 1):S57-S60.

2. Rogers HD, Macgregor JL, Nord KM, et al. Acquired epidermodysplasia verruciformis. J Am Acad Dermatol. 2009;60:315-320.

3. Henley JK, Hossler EW. Acquired epidermodysplasia verruciformis occurring in a renal transplant recipient. Cutis. 2017;99:E9-E12.

4. Jacyk WK, De Villiers EM. Epidermodysplasia verruciformis in Africans. Int J Dermatol. 1993;32:806-810.

5. Fox SH, Elston DM. Epidermodysplasia verruciformis and the risk for malignancy. Cutis. 2016;98:E10-E12.

6. Shruti S, Siraj F, Singh A, et al. Epidermodysplasia verruciformis: three case reports and a brief review. Acta Dermatovenerol Alp Pannonica Adriat. 2017;26:59-61. 